\title{
Flufenamic acid prevents behavioral manifestations of salicylate-induced tinnitus in the rat
}

\author{
Ramazan Bal ${ }^{1}$, Yasemin Ustundag ${ }^{2}$, Funda Bulut ${ }^{3}$, Caner Feyzi Demir ${ }^{4}$, Ali Bal ${ }^{5}$
}

\author{
${ }^{1}$ Department of Physiology, Faculty of Medicine, Gaziantep University, Gaziantep, \\ Turkey \\ 2Department of Anatomy, Faculty of Veterinary, Firat University, Elazig, Turkey \\ ${ }^{3}$ Department of Medical Biology, Faculty of Medicine, Kirikkale University, Kirikkale, \\ Turkey \\ ${ }^{4}$ Department of Neurology, Faculty of Medicine, Firat University, Elazig, Turkey \\ ${ }^{5}$ Department of Plastic-Reconstructive and Esthetic Surgery, Faculty of Medicine, \\ Firat University, Elazig, Turkey
}

Submitted: 7 March 2014

Accepted: 21 May 2014

Arch Med Sci 2016; 12, 1: 208-215

DOI: 10.5114/aoms.2016.57597

Copyright @ 2016 Termedia \& Banach

\section{Abstract}

Introduction: Tinnitus is defined as a phantom auditory sensation, the perception of sound in the absence of external acoustic stimulation. Given that flufenamic acid (FFA) blocks TRPM2 cation channels, resulting in reduced neuronal excitability, we aimed to investigate whether FFA suppresses the behavioral manifestation of sodium salicylate (SSA)-induced tinnitus in rats. Material and methods: Tinnitus was evaluated using a conditioned lick suppression model of behavioral testing. Thirty-one Wistar rats, randomly divided into four treatment groups, were trained and tested in the behavioral experiment: (1) control group: DMSO + saline $(n=6)$, (2) SSA group: DMSO + SSA $(n=6)$, (3) FFA group: FFA $(66 \mathrm{mg} / \mathrm{kg}$ bw $)+$ saline $(n=9)$, (4) FFA + SSA group: FFA $(66 \mathrm{mg} / \mathrm{kg} \mathrm{bw})+\mathrm{SSA}(400 \mathrm{mg} / \mathrm{kg}$ bw) $(n=10)$. Localization of TRPM 2 to the plasma membrane of cochlear nucleus neurons was demonstrated by confocal microscopy.

Results: Pavlovian training resulted in strong suppression of licking, having a mean value of $0.05 \pm 0.03$ on extinction day 1 , which is below the suppression training criterion level of 0.20 in control tinnitus animals. The suppression rate for rats having both FFA ( $66 \mathrm{mg} / \mathrm{kg}$ bw) and SSA ( $400 \mathrm{mg} / \mathrm{kg} \mathrm{bw})$ injections was significantly lower than that for the rats having SSA injections $(p<0.01)$.

Conclusions: We suggest that SSA-induced tinnitus could possibly be prevented by administration of a TRPM2 ion channel antagonist, FFA at $66 \mathrm{mg} / \mathrm{kg}$ bw.

Key words: tinnitus, salicylate, flufenamic acid, TRPM2, experimental, rat.

\section{Introduction}

Tinnitus is defined as a condition or phantom sensation rather than a disease, which is perceived as roaring, buzzing, hissing, whistling and ringing in the ear, due to altered spontaneous neural activity without an external acoustic stimulus $[1,2]$. It is usually a consequence of drug toxicity or acoustic trauma [3-6]. It is estimated that $5-15 \%$ of the general population and western population experience this condition [1, 7]. Extending from intermittent form to persistent form, tinnitus changes the quality of daily life. Its effect ranges from a minor to a major influ-

\author{
Corresponding author: \\ Prof. Dr. Ramazan Bal \\ Department of Physiology \\ Faculty of Medicine \\ Gaziantep University \\ 27310 Gaziantep, Turkey \\ Phone: +90342 \\ 3606060/77732 \\ Fax: +90 3423601617 \\ E-mail: rbal@gantep.edu.tr, \\ ramazanbal1969@gmail.com
}


ence, preventing spatial learning and intellectual working $[7,8]$. Therefore, patients may be led to social isolation because of anxiety, insomnia and depression, and even to commit suicide [8].

Flufenamic acid (FFA) is a member of fenamates, which are derived from $\mathrm{N}$-phenylanthranilic acid and in the group of non-steroidal anti inflammatory drugs [9-11]. Fenamates, including FFA, cause inhibition in pain pathways, inflammation and pyrexia by reducing prostaglandin synthesis and inhibiting cyclooxygenase (COX) enzyme, especially in the treatment of joint and musculoskeletal disorders [9, 11-14]. Additionally, FFA is known to have an antagonistic effect on TRPM2 ion channels $[15,16]$. TRPM2 is a $\mathrm{Ca}^{2+}$-permeable, non-selective member of the transient receptor potential melastatin family of cation channels. TRPM2 channels are activated by reactive oxygen/nitrogen species (ROS/RNS) and ADP-ribose (ADPR) [15, 17]. TRPM2 has been shown to be highly expressed in the central nervous system (CNS) including the midbrain $[18,19]$.

The treatment options of tinnitus in clinical practice are very limited, although there have been many drug treatments suggested, including anticonvulsants, antidepressants, GABAergic and antiglutamatergic agents, antidopaminergic agents, and antiepileptic drugs, as reviewed by Langguth and Salvi [5], Fioretti and Eibenstein [6], and Noble [20]. Deep brain stimulation [21], as in the case of epilepsy [22], has also been reported to give some relief. But they, contrary to their claims, are not used in clinics, since the FDA has not approved any of these therapeutic approaches to treat tinnitus.

Because tinnitus is commonly thought to be associated with hyperexcitability of neurons in the central auditory system, including the cochlear nucleus (CN), the inferior colliculus and the auditory cortex [12, 23, 24], blocking TRPM2 cation channels by administering FFA would modulate neural activity of the neurons in the central auditory pathway, resulting in a decrease of the neuronal excitability in auditory nuclei. The decreased neural excitability, in turn, is expected to suppress behavioral signs of tinnitus.

Therefore, we aimed to investigate the effects of FFA as a blocker of TRPM2 cation channels in a sodium salicylate (SSA)-induced tinnitus animal model.

\section{Material and methods}

\section{Animals}

Thirty-one healthy adult female Wistar albino rats, aged 8-9 weeks and with weight in the range of 180-210 g, were obtained from and maintained in Firat University Experimental Research Centre (Elazig, Turkey). They were housed in groups of six and kept at room temperature in a $12 \mathrm{~h}: 12 \mathrm{~h}$ dark-light cycle schedule. Animals were fed with a balanced commercial diet (Elazig Food Company, Elazig, Turkey) ad libitum, but were water deprived throughout the behavioral testing period. Their weights were measured and recorded daily during the experimental period. Approval for the whole experimental process was obtained from Firat University Animal Experimentation Ethics Committee.

Thirty-one Wistar rats, randomly divided into four treatment groups, were trained and tested in the behavioral experiment: (1) control group: DMSO + saline $(n=6),(2)$ SSA group: DMSO + SSA $(n=6)$, (3) FFA group: FFA (66 mg/kg bw) + saline $(n=9)$, (4) FFA + SSA group: FFA $(66 \mathrm{mg} / \mathrm{kg} \mathrm{bw})+$ SSA $(400 \mathrm{mg} / \mathrm{kg} \mathrm{bw})(n=10)$.

\section{Drugs}

Sodium salicylate and FFA were purchased from Sigma. Sodium salicylate solution was prepared freshly in normal saline solution on the day of administration and was administered subcutaneously at $400 \mathrm{mg} / \mathrm{kg}$ (in a $100 \mathrm{mg} / \mathrm{ml}$ solution of saline). This dose has been shown to evoke behavioral manifestation of tinnitus [25]. Sodium salicylate or saline was injected $2 \mathrm{~h}$ before the testing session. Flufenamic acid was dissolved as a $1 \mathrm{M}$ sol in dimethyl sulfoxide (DMSO), and FFA or vehicle (DMSO) was injected intraperitoneally $5 \mathrm{~h}$ before the testing session. In order to determine the doses of FFA to be tested, a pilot study was first conducted in untrained animals, which were deprived of water for $18 \mathrm{~h}(n=6)$. Flufenamic acid at $66 \mathrm{mg} / \mathrm{kg}$ bw had no apparent effects on their movements, feeding and drinking behavior, alertness and sustained attention to the environment. This dosage of FFA constitutes an appropriate concentration of FFA in body fluids for blockage of TRPM 2 channels.

\section{Conditioning chamber}

Training and assessment of each subject was conducted in the same chamber for all sessions, which were conducted at the same time each day. Assessment of tinnitus was carried out in a homemade conditioning system. The conditioning chamber $(40 \mathrm{~cm} \times 30 \mathrm{~cm} \times 45 \mathrm{~cm})$ was housed in a sound-attenuating surrounding box $(80 \mathrm{~cm}$ $\times 45 \mathrm{~cm} \times 60 \mathrm{~cm}$, minimal attenuation of $45 \mathrm{~dB}$ at $10 \mathrm{kHz}$ ). The conditioning system consisted of a tungsten bar floored cage, sound generator, camera, apparatus for producing electric shocks and remote controls for sound and electric shock generators, and a computer. Videos were recorded by a camera (Microsoft, HD5000) which was located on the most appropriate position at the left 
side of the cage. A drinking bottle was positioned $5 \mathrm{~cm}$ above the floor, which can be seen best by the camera positioned at the left hand side of the cage. The illumination of the system was provided by a house light, which was placed on the ceiling of the cage. The house lights within the chamber were low-wattage bulbs, so there were no audible background sounds in the chamber due to the illumination. The current shock stimuli for Pavlovian conditioning training was generated by a constant current shock source $(0.5 \mathrm{~mA}, 1 \mathrm{~s})$ and delivered by tungsten bars $(4 \mathrm{~mm})$ which were lined on the floor with $1.0 \mathrm{~cm}$ spaces. A pure tone $(10 \mathrm{kHz}, 65 \mathrm{~dB})$ was turned on before placing the animal in the conditioning chamber and delivered during the 30-min testing session. The sound was delivered to the conditioning chamber by a speaker which was located on the ceiling of the cage. Licking number for each animal was determined by the observation of the videos in slow motion, which was taken during the assessment.

\section{Training and experimental testing}

In the present study, a behavioral conditioning paradigm was used, which is based on the original model of Jastreboff et al. [4]. For the aim of demonstrating phantom sound sensation caused by SSA, the animals were subjected to a procedure consisting of three phases: initial lick training, Pavlovian conditioned licking suppression, and extinction, which are based on the study of Jastreboff et al. [4]. The animals were weighed and deprived of water the day prior to behavioral testing and then allowed to drink continuously during the experimental session with a constant background sound $(10 \mathrm{kHz}, 65 \mathrm{~dB})$ produced by a sound generator (Sine/Square Wave Generator, Dick Smith Electronics, NZ) as a safety signal. All acoustic stimuli were calibrated using a high precision sound level meter (Sensonic, Turkey). In addition, the animals were exposed to the same background sound continuously in their home environment. In the initial lick training part the animals were familiarized with the cage, water bottle and silent environment. Initial lick training lasted for 2 days. For the initial lick training, the pure tone was turned on before placing the animal in the conditioning chamber and delivered during the 30-min testing session. Four randomly chosen 30 s silent intervals were given at the 30-min testing session (conditioned stimulus, CS). Thirty-second offset of background noise at random intervals was centered around times approximately 5 , 10, 19 and 28 min after the start of the session. The exact timing of the background noise's offset varied randomly across experimental days. The animals were then subjected to the Pavlovian conditioned licking suppression phase, in which $30 \mathrm{~s}$ offset of background noise was coupled with electrical foot shocks (unconditioned stimulus, US). After 5 sessions, animals learned well the association between the offsetting of the background sound and foot shocks, and therefore in response to offset of the background sound the animals stopped licking, namely drinking water. When the suppression rate (SR) falls below 0.2 , the lick suppression phase is considered to be established [25]. After lick suppression was established, the animals were subjected to the extinction protocol with one trial per day for 4 days using the same procedure as in the Pavlovian suppression training, for which no foot shock was delivered during 30 s offset of background noise.

For tinnitus induction, the animals were given a single injection of SSA ( $400 \mathrm{mg} / \mathrm{kg}$, sc). Sodium salicylate treatment is well known to cause tinnitus-like behavior in rats [25]. Assessment began $2 \mathrm{~h}$ after SSA administration. The rats with tinnitus $2 \mathrm{~h}$ after SSA injection would be expected to behave always as though the tone was present, even in its absence, since tinnitus would prevent them from sensing the CS, the offset of background noise, and therefore the animals would keep licking. If the SR was above 0.4, the animals were identified as having tinnitus [25].

Suppression of the drinking activity during the offset of background noise was measured by calculating SR, for which licking numbers of animals were counted during the tone off period (Ntoneoff: offset of background noise, silence) and the tone on period (Ntoneon: presence of background noise) periods. $\mathrm{SR}=($ Ntoneoff $/ 2 \mathrm{~min}) /[$ (Ntoneoff/2 $\mathrm{min})+($ Ntoneon/28 $\mathrm{min})]$.

Suppression rates of the 31 animals before the behavioral training were measured to be between 0.403 and 0.709 . At the end of evaluation, if their SR was equal to or greater than 0.4, animals were considered still to have tinnitus; but if it was less than 0.2 , tinnitus was considered to be eliminated.

\section{Immunohistochemistry}

Mice aged 8 weeks old were decapitated under ether anesthesia and brain stems containing $\mathrm{CN}$ were dissected, and immediately fixed in ice cold $4 \%$ paraformaldehyde solution made up in $0.1 \mathrm{M}$ phosphate buffer (PB; $\mathrm{pH}: 7.4$ ) for $30 \mathrm{~min}$. The tissues were transferred to ice-cold $30 \%$ sucrose in $0.1 \mathrm{M}$ phosphate buffer with $0.1 \% \mathrm{Na}$ azide for cryoprotection overnight.

The brain stems were cut on a freezing microtome with temperature adjusted to $-20^{\circ} \mathrm{C}$ at the level of the $\mathrm{CN}$ into a series of adjacent $20 \mu \mathrm{m}$ thick coronal sections and were then thaw-mounted on polylysine-coated glass slides. Sections were incubated for at least $2 \mathrm{~h}$ in a blocking solution that contained $0.1 \%$ Triton $\mathrm{X}, 0.1 \%$ sodium azide, 
$3 \%$ bovine serum albumin, and 5\% normal goat serum in PBS. Tissue was incubated overnight at $+4^{\circ} \mathrm{C}$ in the following primary antibodies: (i) microtubule-associated proteins (MAP2) (1: 1000 mouse anti-MAP2 monoclonal, Sigma), (ii) TRPM2 (1: 100 rabbit anti- TRPM2 monoclonal, Abcam).

After rinsing with PBS, the sections were incubated for $2 \mathrm{~h}$ at room temperature at a $1: 1000$ dilution in PBS including 3\% donkey serum, 3\% bovine serum albumin, 0.1\% Tween-20 and 0.1\% sodium azide with the following relevant secondary antibodies: donkey $\alpha$-rabbit, 488, donkey $\alpha$-mouse IgG 555 (Alexa fluor, Invitrogen, Carlsbad, CA, USA). The sections were then rinsed with PBS. Images were captured using a scanning confocal microscope (LSM5, Zeiss). We recorded both single optical sections and image stacks (z-step $0.14 \mu \mathrm{m})$ that consisted of 6-15 image planes. Labeling with MAP2 was performed to recognize the substructures of CN.

\section{Antibody specificity}

The antibodies were tested for their specificities by the manufacturers. To confirm non-specific immunolabelling using the immunofluorescence method, primary antibodies were excluded but the secondary antibody steps were conducted to completion. In that case, no cross-reactivity or significant background staining was seen (not shown).

\section{Statistical analysis}

Behavioral data were analyzed using one-way ANOVA and post hoc Tukey's test. Values were considered statistically significant if $p<0.05$. Results are presented as mean \pm SEM. The SPSS/ PC program (Version 10.0; SPSS, Chicago, IL) was used for the statistical analysis.

\section{Results}

A preliminary screening was performed on the second day of the initial lick training to identify those animals that were well responsive to the 30 s offsets of background noise by showing any behavioral signs of noticing the tone-off period. The screening revealed that 29 of 60 rats were not responsive to the $30 \mathrm{~s}$ offsets of background noise. Therefore, those 29 animals that were not responsive to the tone-off period were excluded from the study.

The persistence of the training's effect was studied in the rats in Vehicle + Saline groups subjected to the procedure of initial lick training and Pavlovian conditioned licking suppression, and extinction without any treatment of SSA or FFA throughout 4 days. The suppression ratios for vehicle + saline and vehicle + SSA groups are comparatively illustrated in Figure 1. Pavlovian training resulted in strong suppression of licking, having a mean value of $0.05 \pm 0.03$ on extinction day 1 , which is below the suppression training criterion level of 0.20 . Note that the SR was below 0.20 on the training day first and second days of extinction. However, the SR then increased gradually. The behavioral response was extinguished due to the removal of the shock.

As can be seen in Figure 1, vehicle + SSA groups had higher suppression rates throughout extinction days 1-4 compared to the vehicle + saline group. The SSA group had a suppression rate of $0.57 \pm 0.04$ on extinction day 1 . The differences between the suppression rate for vehicle + SSA and vehicle + saline groups were significant for extinction days 1 and 2 .

The mean SR for rats treated with SSA was significantly higher than that for the rats treated with only vehicle and saline $(p<0.01)$ for not only extinction day 1 but also extinction day 2 . But there were no significant differences on extinction days 3 and $4(p>0.05)$. The SSA-treated animals on extinction days 1 and 2 perceived the tones as 'noisy' due to presence of tinnitus as a safety signal to drink water.

Mean suppression rates for vehicle + saline, vehicle + SSA, FFA + saline and FFA + SSA groups on extinction day 1 are illustrated in Figure 2 as a bar graph. There was no significant difference between the suppression rates for vehicle + saline and FFA + saline groups. This suggests that FFA did not induce tinnitus-like behavior in these animals. The suppression rate for rats having both FFA and SSA injections was significantly lower than that for the rats having SSA injections $(p<0.01)$, suggesting that FFA ameliorated tinnitus-like be-

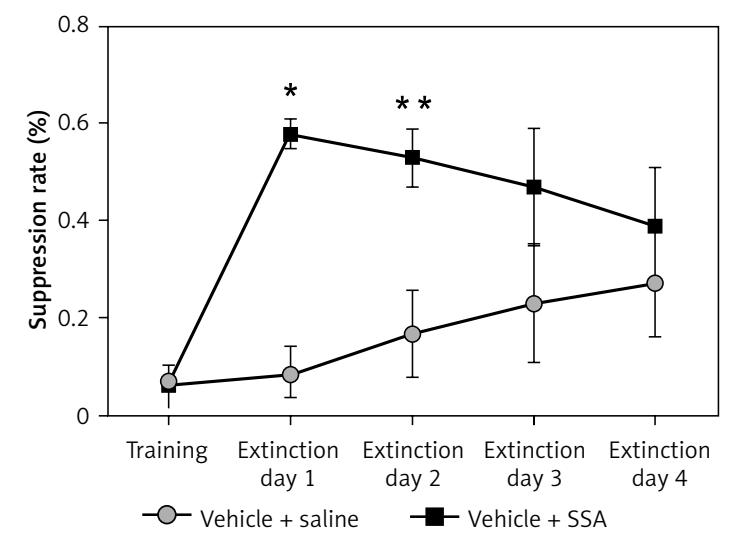

Figure 1. Suppression rates for vehicle + saline and vehicle + SSA groups on training and extinction days $1-4$. Note that in the saline + vehicle group the suppression rate was below the suppression training criterion level of 0.20 . But the suppression rate in the SSA-treated group increased much above the suppression training criterion level of 0.20

${ }^{*} p<0.001,{ }^{* *} p<0.001$. 


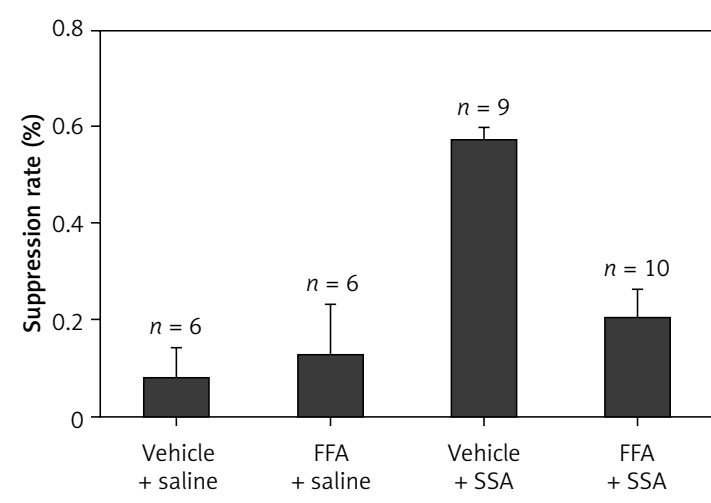

Figure 2. Suppression rates for vehicle + saline, vehicle + SSA, FFA + saline and FFA + SSA groups on extinction day 1 . Note that FFA reduced the suppression rate increased by SSA to below the suppression training criterion level of 0.20

havior in these animals. There was no significant difference between the vehicle + saline group and the FFA + SSA group.

The protein expression and distribution patterns of TRPM2 were examined using subtypespecific antibodies. Both the soma and dendrites of neurons of the anterioventral cochlear nucleus (AVCN) were occasionally labeled, but the stainings cannot certainly be associated with particular cell types (Figure 3).

\section{Discussion}

To our knowledge, this is the first study investigating therapeutic effects of FFA as a TRPM2 ion channel antagonist on SSA-induced tinnitus in rats. The major finding of the present study is that FFA at a dose of $66 \mathrm{mg} / \mathrm{kg}$ bw had a significant therapeutic effect on tinnitus.

It is well known from previous studies that salicylate administration at doses over $150 \mathrm{mg} / \mathrm{kg}$ bw induces tinnitus in humans $[26,27]$ and in animals [28]. Sodium salicylate -induced tinnitus is the most widely used animal model to study tinnitus in rats $[4,5,26,29]$. As expected from salicylate injection (400 mg/kg bw) [25, 29, 30], animals treated with salicylate showed tinnitus-like behavior, as evident by a significantly higher suppression rate on extinction day 1 compared to the DMSO + saline group (Figure 1). Introduction of an internally generated continuous auditory signal induced by salicylate clearly resulted in faster extinction because auditory phantom perception induced by SSA is sufficiently similar to the background noise in the DMSO + SSA group (Figure 1). As reported previously [29], this tinnitus-like behavior was reduced by 2 days after a single salicylate treatment (400 mg/kg bw, sc) in the DMSO + SSA group.

The neuronal mechanisms of phantom auditory sound perception induced by salicylate are still obscure. However, it is widely accepted that tinni- tus results from an increase in spontaneous activity of neurons in the auditory system. It has been demonstrated that exposure to acoustic trauma, which induces tinnitus in humans, causes increas es in spontaneous activity of neurons in the dorsal cochlear nucleus (DCN) [7, 23, 31-33]. Salicylate is also known to increase spontaneous activity in the central auditory system [34].

In the present study it was hypothesized that blocking TRPM2 ion channels would modulate neural activity in the central auditory pathway through decreasing the neuronal excitability, resulting in an increase in inhibitory signals in auditory nuclei, which leads to decreases of tinnitus generating signals. Consistently, we demonstrated in this study that the suppression rate for rats treated with both FFA and SSA was significantly lower than that for the rats having SSA injections $(p<0.01)$. Namely, application of FFA, an antagonist of TRPM2 cation channels, prevents SSA-induced tinnitus, suggesting that TRPM2 ion channels may partly be involved in the induction of tinnitus.

However, flufenamic acid has been suggested to be a modulator of some other ion channels, including non-selective cation channels, chloride channels, potassium, calcium and sodium channels, as well as two-pore outwardly rectifying potassium channels [35]. Therefore, given the broad spectrum of its effects on ion channels, the underlying mechanism of its effects on tinnitus behavior could also be due to modulations of other ions. It is possible that FFA might upregulate the ion channels that cause hyperpolarization, such as potassium ion channels, or might downregulate the ion channels that cause depolarization.

As suggested previously [32], there is a delicate balance between inhibition and excitation in the central auditory system for normal auditory perception. In the auditory pathway, decreased inhibition/increased excitation, resulting in hyperexcitability of neurons, constitutes an important mechanism of tinnitus pathology [31, 36, 37]. Namely, in the case of tinnitus, the balance is lost in favor of excitation either due to decreased inhibition or increased excitation [38, 39]. Whatever the cause of tinnitus, it is likely that inhibition of TRPM 2 by FFA might possibly change the balance between inhibition and excitation in favor of inhibition, since the activation of TRPM 2 cation channels causes the neuron to depolarize [40].

TRPM 2 has been shown to be highly expressed in the brain $[16,18,19,37]$. More specifically, immunohistochemical staining and PCR studies show that neurons in the cochlear nucleus and inferior colliculus, auditory nuclei, possess TRPM2 ion channels (Figure 3).

In addition to its anti-inflammatory effects, FFA is now well known to be a blocker of TRPM 2 cation 

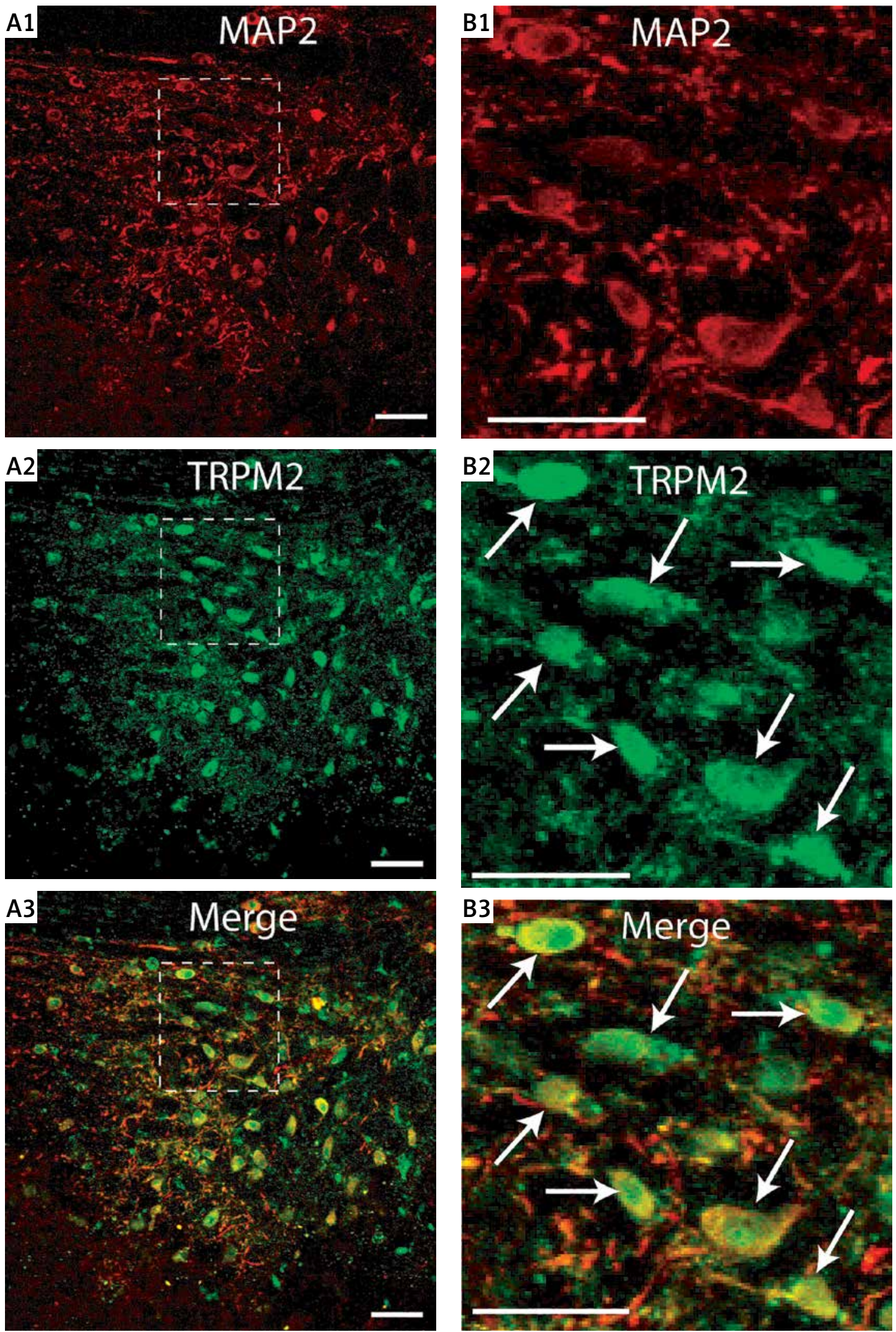

Figure 3. Confocal images of mouse AVCN sections immunolabeled with TRPM2. The small area in A1, A2 and A3 indicated with a dashed rectangular box is magnified in B1, B2 and B3. Sections were double labeled with MAP2 (A1 and B1) as well as TRPM2 channel (A2 and B2). The AVCN substructure of CN was identified by the staining of MAP2 labeling. The arrows show the cell bodies stained with TRPM2 in B2 and B3. Note that the staining was intense. Calibration bar, $40 \mu \mathrm{m}$ 
channels [16]. Flufenamic acid appears to have an ameliorating effect on tinnitus, possibly through its blocking action of TRPM2 ion channels. However, there might also be other possible mechanisms whereby FFA exerts its therapeutic effect on tinnitus, since FFA has some other effects such as blocking action on connexins [14, 41], inhibition of $\mathrm{Ca}^{2+}$-activated chloride currents [42], modulation of GABAA receptors $[24,43]$, and blocking action on voltage-gated $\mathrm{Na}^{+}$channels $[44,45]$ and the calcium-activated cationic channel [46].

It should be noted that SSA-induced tinnitus is an acute form of tinnitus and usually reversible. Thus it needs no treatment. However, treatment of SSA-induced tinnitus with FFA in experimental animals might lead to a suggestion that FFA should also help to prevent tinnitus caused by other factors, such as pathological changes, noise exposure, head trauma, ageing and side effects of clinical medicine other than salicylate in humans.

In conclusion, in the present study, we demonstrated that FFA administration to rats having tinnitus induced by SSA resulted in significantly lower suppression rates compared to those for rats with tinnitus induced by SSA $(p<0.01)$. Therefore, we suggest that SSA-induced tinnitus could possibly be prevented by administration of a TRPM2 ion channel antagonist, FFA, at $66 \mathrm{mg} / \mathrm{kg}$ bw. However, further investigations are necessary in order to understand better the therapeutic potential of FFA in tinnitus.

\section{Acknowledgments}

The immunohistochemical staining part of the study was supported by the Scientific and Technical Research Council of Turkey (TUBITAK) under Grant No. SBAG-110S397.

\section{Conflict of interest}

The authors declare no conflict of interest.

\section{References}

1. Vanneste S, Heyning PV, Ridder DD. Contralateral parahippocampal gamma-band activity determines noiselike tinnitus laterality: a region of interest analysis. Neuroscience 2011; 199: 481-90.

2. Zheng Y, Vagal S, McNamara E, Darlington CL, Smith PF. A dose-response analysis of the effects of $L$-baclofen on chronic tinnitus caused by acoustic trauma in rats. Neuropharmacology 2012; 62: 940-6.

3. Brown RD, Penny JE, Henley CM, et al. Ototoxic drugs and noise. Ciba Foundation Symposium 1981; 85: 151-71.

4. Jastreboff PJ, Brennan JF, Coleman JK, Sasaki CT. Phantom auditory sensation in rats: an animal model for tinnitus. Behav Neurosci 1988; 102: 811-22.

5. Langguth B, Salvi R, Elgoyhen AB. Emerging pharmacotherapy of tinnitus. Exp Opin Emerg Drugs 2009; 14: 687-702.
6. Fioretti A, Eibenstein A, Fusetti M. New trends in tinnitus management. Open Neurol J 2011; 5: 12-7.

7. Schecklmann M, Landgrebe M, Poeppl TB, et al. Neural correlates of tinnitus duration and distress: a positron emission tomography study. Hum Brain Mapp 2013; 34: 233-40.

8. Mao JC, Pace E, Pierozynski P, et al. Blast-induced tinnitus and hearing loss in rats: behavioral and imaging assays. J Neurotrauma 2012; 29: 430-44.

9. Aly FA, Al-Tamimi SA, Alwarthan AA. Determination of phenolic sympathomimetic drugs in pharmaceutical samples and biological fluids by flow-injection chemiluminescence. J AOAC Int 2000; 83: 1299-305.

10. Hu H, Tian J, Zhu Y, et al. Activation of TRPA1 channels by fenamate nonsteroidal anti-inflammatory drugs. Pflugers Arch 2010; 459: 579-92.

11. Zhao ZG, Zhang $M$, Zeng XM, et al. Flufenamic acid bi-directionally modulates the transient outward $\mathrm{K}(+)$ current in rat cerebellar granule cells. J Pharmacol Exp Ther 2007; 322: 195-204.

12. Coyne L, Su J, Patten D, Halliwell RF. Characterization of the interaction between fenamates and hippocampal neuron GABA(A) receptors. Neurochem Int 2007; 51: 440-6.

13. Fernandez M, Lao-Peregrin C, Martin ED. Flufenamic acid suppresses epileptiform activity in hippocampus by reducing excitatory synaptic transmission and neuronal excitability. Epilepsia 2010; 51: 384-90.

14. Gardam KE, Geiger JE, Hickey CM, Hung AY, Magoski NS. Flufenamic acid affects multiple currents and causes intracellular Ca2+ release in Aplysia bag cell neurons. J Neurophysiol 2008; 100: 38-49.

15. Naziroglu M. TRPM2 cation channels, oxidative stress and neurological diseases: where are we now? Neurochem Res 2011; 36: 355-66.

16. Hill K, Benham CD, McNulty S, Randall AD. Flufenamic acid is a pH-dependent antagonist of TRPM2 channels. Neuropharmacology 2004; 47: 450-60.

17. Takahashi N, Kozai D, Kobayashi R, Ebert M, Mori Y. Roles of TRPM2 in oxidative stress. Cell Calcium 2011; 50: 279-87.

18. Kunert-Keil C, Bisping F, Kruger J, Brinkmeier H. Tissue-specific expression of TRP channel genes in the mouse and its variation in three different mouse strains. BMC Genomics 2006; 7: 159.

19. Kraft R, Harteneck C. The mammalian melastatin-related transient receptor potential cation channels: an overview. Pflugers Arch 2005; 451: 204-11.

20. Noble W. Treatments for tinnitus. Trends Amplif 2008; 12: 236-41.

21. Cheung SW, Larson PS. Tinnitus modulation by deep brain stimulation in locus of caudate neurons (area LC). Neuroscience 2010; 169: 1768-78.

22. Tykocki T, Mandat T, Kornakiewicz A, Koziara H, Nauman P. Deep brain stimulation for refractory epilepsy. Arch Med Sci 2012; 8: 805-16.

23. Kaltenbach JA, Godfrey DA, Neumann JB, McCaslin DL, Afman CE, Zhang J. Changes in spontaneous neural activity in the dorsal cochlear nucleus following exposure to intense sound: relation to threshold shift. Hear Res 1998; 124: 78-84.

24. Maksay G, Korpi ER, Uusi-Oukari M. Bimodal action of furosemide on convulsant [3H]EBOB binding to cerebellar and cortical GABA(A) receptors. Neurochem Int 1998; 33: 353-8.

25. Kizawa K, Kitahara T, Horii A, et al. Behavioral assessment and identification of a molecular marker in a salicylate-induced tinnitus in rats. Neuroscience 2010; 165 : 1323-32. 
26. Myers EN, Bernstein JM. Salicylate ototoxicity: a clinical and experimental study. Arch Otolaryngol 1965; 82: 483-93.

27. McFadden D, Plattsmier HS, Pasanen EG. Aspirin-induced hearing loss as a model of sensorineural hearing loss. Hear Res 1984; 16: 251-60.

28. Bauer CA, Brozoski TJ, Rojas R, Boley J, Wyder M. Behavioral model of chronic tinnitus in rats. Otolaryngol Head Neck Surg 1999; 121: 457-62.

29. Zheng Y, Hooton K, Smith PF, Darlington CL. Carbamazepine reduces the behavioural manifestations of tinnitus following salicylate treatment in rats. Acta Otolaryngol 2008; 128: 48-52.

30. Zheng Y, Stiles L, Hamilton E, Smith PF, Darlington CL. The effects of the synthetic cannabinoid receptor agonists, WIN55,212-2 and CP55,940, on salicylate-induced tinnitus in rats. Hear Res 2010; 268: 145-50.

31. Bauer CA, Turner JG, Caspary DM, Myers KS, Brozoski TJ. Tinnitus and inferior colliculus activity in chinchillas related to three distinct patterns of cochlear trauma. J Neurosci Res 2008; 86: 2564-78.

32. Wang HT, Luo B, Zhou KQ, XU TL, Chen L. Sodium salicylate reduces inhibitory postsynaptic currents in neurons of rat auditory cortex. Hear Res 2006; 215: 77-83.

33. Zhang JS, Kaltenbach JA. Increases in spontaneous activity in the dorsal cochlear nucleus of the rat following exposure to high-intensity sound. Neurosci Lett 1998; 250: 197-200.

34. Jastreboff PJ, Sasaki CT. Salicylate-induced changes in spontaneous activity of single units in the inferior colliculus of the guinea pig. J Acoust Soc Am 1986; 80: 1384-91.

35. Guinamard R, Simard C, Del Negro C. Flufenamic acid as an ion channel modulator. Pharmacol Therap 2013; 138: 272-84.

36. Kaltenbach JA. The dorsal cochlear nucleus as a contributor to tinnitus: mechanisms underlying the induction of hyperactivity. Progr Brain Res 2007; 166: 89-106.

37. Xie YF, Macdonald JF, Jackson MF. TRPM2, calcium and neurodegenerative diseases. Int J Physiol Pathophysiol Pharmacol 2010; 2: 95-103.

38. Eggermont JJ. Central tinnitus. Auris Nasus Larynx 2003; 30 Suppl: S7-12.

39. Eggermont JJ, Roberts LE. The neuroscience of tinnitus. Trends Neurosci 2004; 27: 676-82.

40. Olah ME, Jackson MF, Li H, et al. Ca2+-dependent induction of TRPM2 currents in hippocampal neurons. J Physiol 2009; 587: 965-79.

41. Juszczak GR, Swiergiel AH. Properties of gap junction blockers and their behavioural, cognitive and electrophysiological effects: animal and human studies. Progr Neuropsychopharmacol Biol Psychiatry 2009; 33: 181-98.

42. Kim SJ, Shin SY, Lee JE, Kim JH, Uhm DY. Ca2+-activated $\mathrm{Cl}$ - channel currents in rat ventral prostate epithelial cells. Prostate 2003; 55: 118-27.

43. Sinkkonen ST, Mansikkamaki S, Moykkynen T, Luddens H, Uusi-Oukari M, Korpi ER. Receptor subtype-dependent positive and negative modulation of GABA(A) receptor function by niflumic acid, a nonsteroidal anti-inflammatory drug. Mol Pharmacol 2003; 64: 753-63.

44. Lee RJ, Shaw T, Sandquist M, Partridge LD. Mechanism of action of the non-steroidal anti-inflammatory drug flufenamate on [Ca2+]i and $\mathrm{Ca}(2+)$-activated currents in neurons. Cell Calcium 1996; 19: 431-8.

45. Lee HM, Kim HI, Shin YK, Lee CS, Park M, Song JH. Diclofenac inhibition of sodium currents in rat dorsal root ganglion neurons. Brain Res 2003; 992: 120-7.
46. Cho H, Kim MS, Shim WS, Yang YD, Koo J, Oh U. Calcium-activated cationic channel in rat sensory neurons. Eur J Neurosci 2003; 17: 2630-8. 